\title{
Typical evanescent and atypical persistent polymorphic cutaneous rash in an adult Brazilian with Still's disease: a case report and review of the literature
}

\author{
Despina Michailidou $^{1} \cdot$ Junghee Shin $^{1} \cdot$ Inga Forde $^{2} \cdot$ Kavitha Gopalratnam $^{1}$ • \\ Paul Cohen $^{3} \cdot$ Angela DeGirolamo $^{1}$
}

Received: 18 June 2015/Accepted: 11 September 2015/Published online: 30 September 2015

(C) The Author(s) 2015

\begin{abstract}
Adult onset Still's disease (AOSD) is a systemic auto-inflammatory condition of unknown etiology, characterized by high fever, an evanescent, salmon-pink maculopapular skin rash, arthralgia or arthritis and leukocytosis. AOSD can also present with atypical cutaneous manifestations, such as persistent pruritic coalescent papules or plaques and linear lesions that have highly distinctive pathological features and are usually associated with severe disease. Herein, we present a 31-year-old Brazilian man with both typical Still's rash and atypical persistent polymorphic cutaneous manifestations associated with severe systemic inflammatory response syndrome. Eosinophils that are consistently lacking in the AOSD-associated skin lesions were evident in the skin biopsy of the persistent atypical cutaneous manifestations and were either drug-related or AOSD-associated.
\end{abstract}

Keywords AOSD $\cdot$ Systemic inflammatory response syndrome $\cdot$ Eosinophils $\cdot$ Atypical cutaneous manifestations

Despina Michailidou

de.michailidou@gmail.com

1 Department of Internal Medicine, Bridgeport Hospital, YaleNew Haven Health System, Bridgeport, CT, USA

2 Section of Pulmonary, Critical Care and Sleep Medicine, Bridgeport Hospital, Yale-New Haven Health System, Bridgeport, CT, USA

3 Department of Pathology, Bridgeport Hospital, Yale University School of Medicine, New Haven, CT, USA

\section{Introduction}

Adult onset Still's disease (AOSD) is a systemic auto-inflammatory condition of unknown etiology, characterized by intermittent spiking high fever, an evanescent, salmonpink or erythematous maculopapular skin rash, arthralgia or arthritis and leukocytosis with at least $80 \%$ neutrophils [1]. Other common symptoms include sore throat, lymphadenopathy, hepatomegaly, and splenomegaly [2]. High serum ferritin levels, elevated ESR and high CRP levels, absent antinuclear antibody (ANA) and rheumatoid factors (RF) are the most common laboratory findings [3, 4]. We report a case of AOSD in a 31-year-old Brazilian man presenting with both typical Still's rash and atypical nonevanescent polymorphic cutaneous manifestations.

\section{Case presentation}

A 31-year-old Brazilian male presented with high quotidian fever and night sweats, non-productive cough, lower back pain and erythematous rash for two weeks. The fever occurred almost daily and ranged from 39 to $40{ }^{\circ} \mathrm{C}$. The rash started from both hands and was characterized by multiple erythematous confluent roundish macules and papules that coalesced to form large, irregular erythematous plaques (Fig. 1). The rash lasted for a few days and then appeared with a different morphology on the flexor surfaces of his arms as an extensive erythematous linear urticarial eruption (Fig. 2a). Subsequently the rash appeared on his upper and lower trunk as multiple intensely pruritic linear urticarial streaks (Fig. 2b). Ibuprofen has been used intermittently to alleviate back pain as well as the fever with minimal relief. He denied any contacts with sick individuals, insect or animal bites and his last trip was 


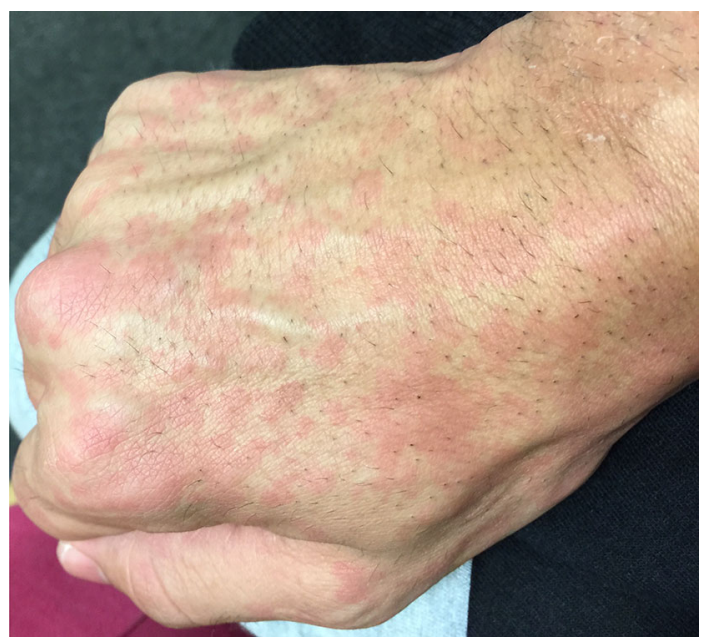

Fig. 1 Typical evanescent rash: Multiple non-pruritic confluent erythematous macules and papules on the dorsal surface of both hands that coalesced to form irregular erythematous plaques
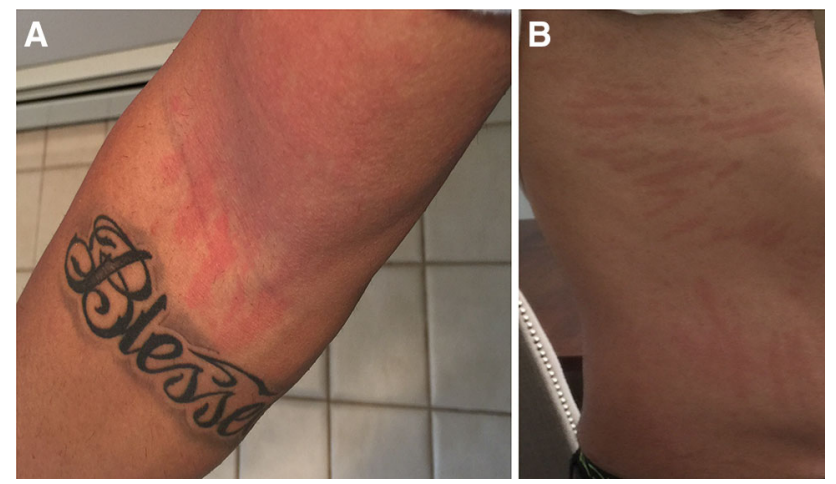

Fig. 2 Atypical urticarial rash: a linear urticarial eruption on the flexor surface of arms. b Multiple intensely pruritic linear urticarial streaks on the upper and lower trunk

to Brazil 10 months ago. He has been sexually active in a monogamous relationship.

On admission, his temperature was $39^{\circ} \mathrm{C}$ and a persistent pigmented plaque $\mathrm{V}$ shaped was evident on his anterior chest extending down the midline to the umbilicus (Fig. 3). Further skin examination revealed a confluent salmon-pink papular eruption on his lower back area and a persistent pigmented plaque on the upper area of his back. Besides mild splenomegaly and a tender right wrist, left second and third proximal interphalangeal joints with no signs of swelling or erythema, the rest of the physical exam was unremarkable. Laboratory profile revealed severe neutrophilic leukocytosis $(30,000$, normal values $4800-10,800 /$ $\mathrm{mcL})$, and a highly elevated serum ferritin levels $(>10,000$, normal values $17.90-464.00 \mathrm{ng} / \mathrm{mL}$ ). Autoantibodies (including ANA, ANCA, RF and anti-CCP) were negative. Blood cultures excluded common viral and bacterial infections and RPR were negative. Serological tests for

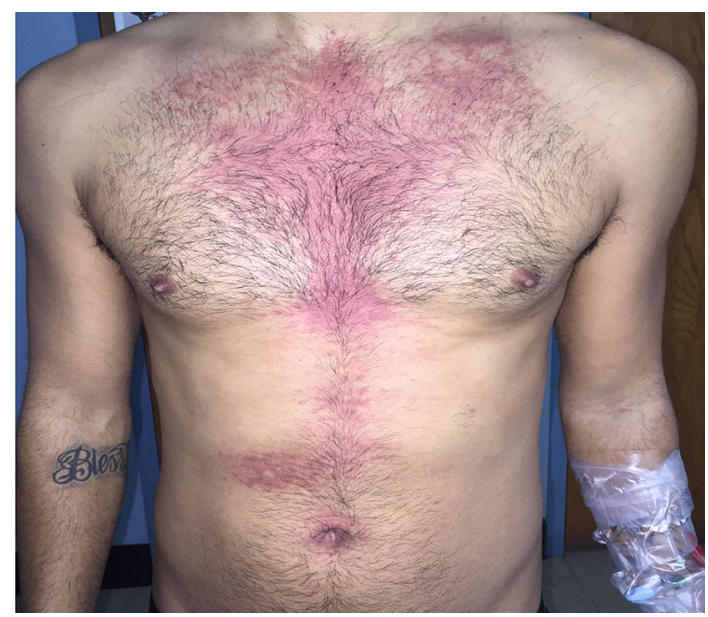

Fig. 3 Atypical persistent rash: persistent pigmented plaque V shaped on the anterior chest extending down the midline to the umbilicus

Hepatitis B and C, HIV, Epstein-Barr and Cytomegalovirus were negative. Borrelia burgdorferi, Bartonella henselae, Rickettsia typhi, RMSF, Typhus and Parvovirus B-19 serologies were negative as well. Parasites for malaria or Babesia microti were undetectable on peripheral blood smear. Transthoracic echocardiogram was negative for vegetations and computed tomography (CT) of the neck, chest and abdomen revealed only borderline mild splenomegaly.

The clinical and laboratory findings were consistent with the diagnosis of AOSD according to Yamaguchi criteria [2]. He was started on $50 \mathrm{mg}$ of prednisone. After 2 weeks of treatment, he returned to our hospital with very high daily spiking fever up to $39.5^{\circ} \mathrm{C}$, perfuse sweating, hypotension, elevated liver enzymes and severe leukocytosis with neutrophil predominance. No new skin lesions were noted. The patient was admitted to intensive care unit due to suspected systemic inflammatory response syndrome and was started on broad-spectrum antibiotics and intravenous fluids. Since the blood cultures were negative, antibiotics were discontinued. Anakinra $100 \mathrm{mg}$ daily subcutaneously was added to $50 \mathrm{mg}$ of prednisone with dramatic resolution of his febrile episodes. The patient was discharged with instructions to gradually taper prednisone.

After 1 week of treatment with Anakinra and while on $40 \mathrm{mg}$ of prednisone the patient remained afebrile but new erythematous plaques appeared on lower abdominal quadrants and a skin biopsy was performed (Fig. 4). Skin biopsy showed a normal epidermis, with an inflammatory infiltrate in the dermis surrounding superficial blood vessels and adnexal structures, and the interstitium as well (Fig. 5a). The inflammatory infiltrate composed of lymphocytes, neutrophils and eosinophils (Fig. 5b). Because of the persistent cutaneous manifestations, the patient was 

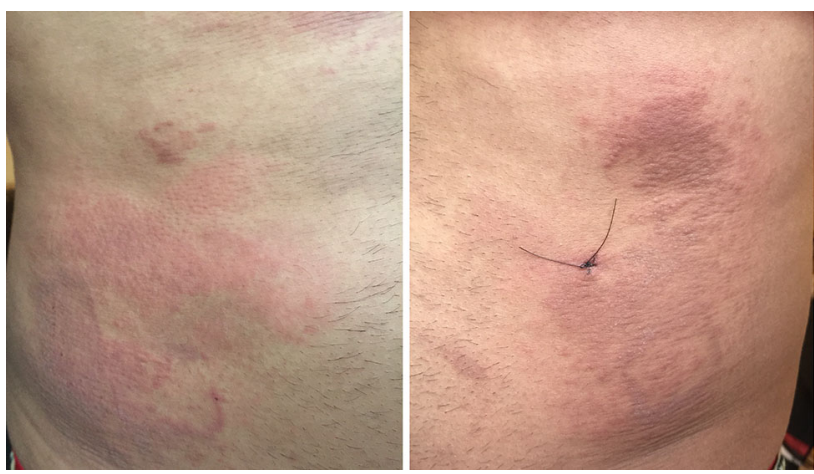

Fig. 4 Atypical persistent pruritic eruption: edematous erythematous plaques on lower abdominal quadrants. Stich denotes the site of skin biopsy

advised to apply on the persistent eruptions of his chest and abdomen triamcinolone cream $0.5 \%$ twice daily. After 1-month follow-up, the skin rash on the above areas almost resolved.

\section{Discussion}

Review of literature, via the PubMed search, using the terms adult onset Still's disease, cutaneous manifestations and eruptions from 1985 to 2014 to retrieve data on the diversity in clinical manifestations and histopathological findings of polymorphic Still's rash, was performed (Table 1). AOSD is a rare young adult systemic autoimmune disorder with diverse clinical manifestations and occasionally unwanted serious organ damage like acute liver failure, adult respiratory distress syndrome, disseminated intravascular coagulation, and hemophagocytic syndrome [5-10]. Thus, early recognition of AOSD is crucial and should be always considered in the differential diagnosis of a systemic inflammatory syndrome, particularly when extensive microbiological workup is negative.
The typical skin rash of AOSD is an evanescent salmonpink non-pruritic or mildly pruritic maculopapular rash, with nonspecific histologic characteristics comprised of a superficial perivascular lymphocytic and scattered neutrophilic infiltrate in the upper epidermis [11-13]. The lesions often develop on the extremities and over the trunk during the peak of the fever and then resolve. AOSD can also present with various atypical cutaneous manifestations and persistent pruritic eruptions (PPEs) are common [14].

PPEs are polymorphic both in morphology and distribution patterns. The more common patterns include lichenoid, linear and dermographism-like eruptions [14], persistent pruritic coalescent papules and plaques [15-17] with linear pigmentation [18], dermal and mucosal hyperpigmentation [19], amyloidosis-like skin eruption [20], generalized peau d'orange appearance of the skin [21], generalized persistent erythema [22], prurigo pigmentosalike eruption [23, 24], vesiculopustules [25], urticaria [26, 27] and fixed papular lesions [28]. The latter are characterized by atypical wheals, present for more than $24-36 \mathrm{~h}$, with symmetrical distribution [29, 30]. Pruritic lesions are usually evident with the presence of linear dermographism from scratching, as was evident in our patient. The most common atypical rash manifestation in AOSD includes the persistent pruritic coalescent papules and plaques and linear lesions [31].

In addition to the typical maculopapular evanescent Still's rash, our patient had also an atypical persistent pigmented eruption manifested with different cutaneous morphology and geographic distribution over his body. The linear urticarial streaky and dermographism-like eruptions on upper extremities and torso sequentially gave place to persistent erythematous plaques on his chest and abdomen. We believe that this type of polymorphic cutaneous eruption may be a predictor of severe systemic inflammatory disease like in our case, and could be associated with activation of macrophages and natural killer cells in

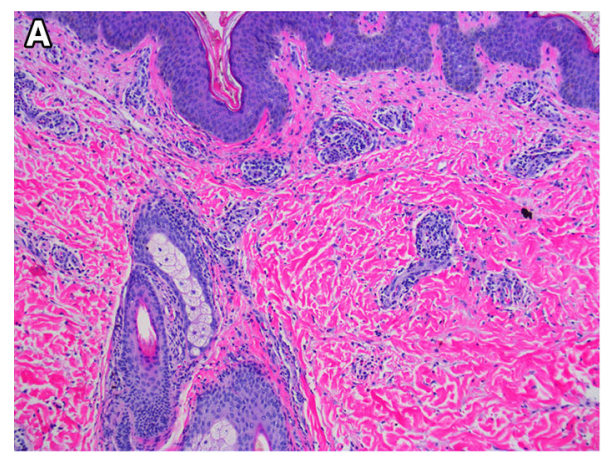

Fig. 5 a Hematoxylin and eosin (H\&E) stain showing periadnexal and perivascular infiltrate of inflammatory cells surrounding blood vessels, hair follicles and the interstitium (in between the vessels and

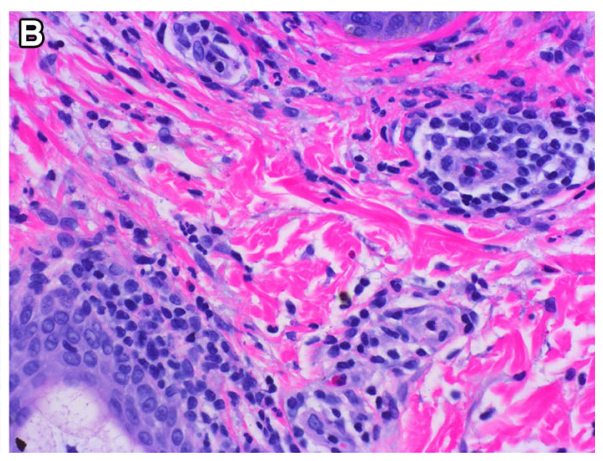

adnexal structures). Original magnification at $\times 100$. b H\&E stain showing perivascular inflammatory infiltration of lymphocytes, neutrophils and eosinophils. Original magnification at $\times 400$ 


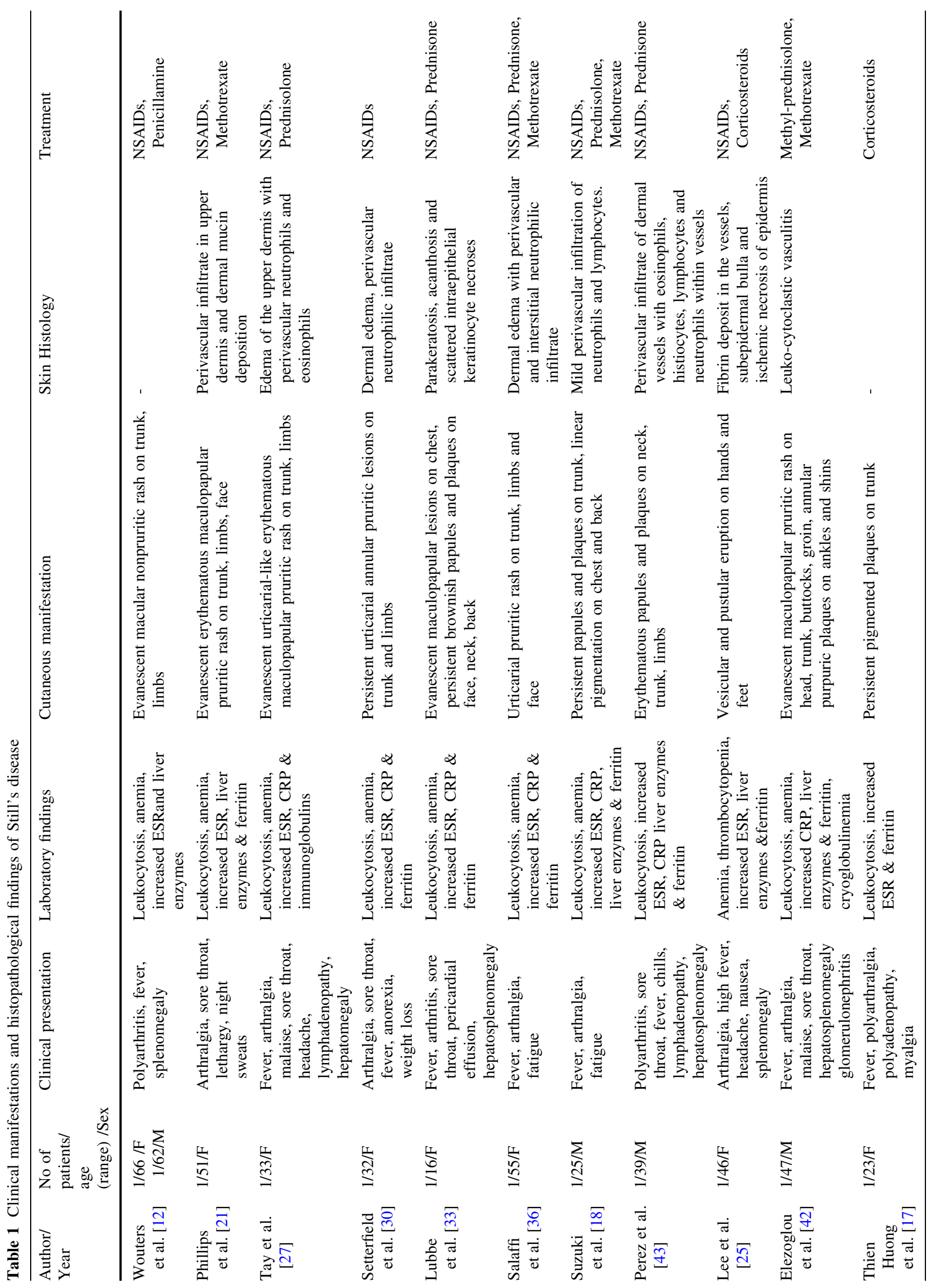




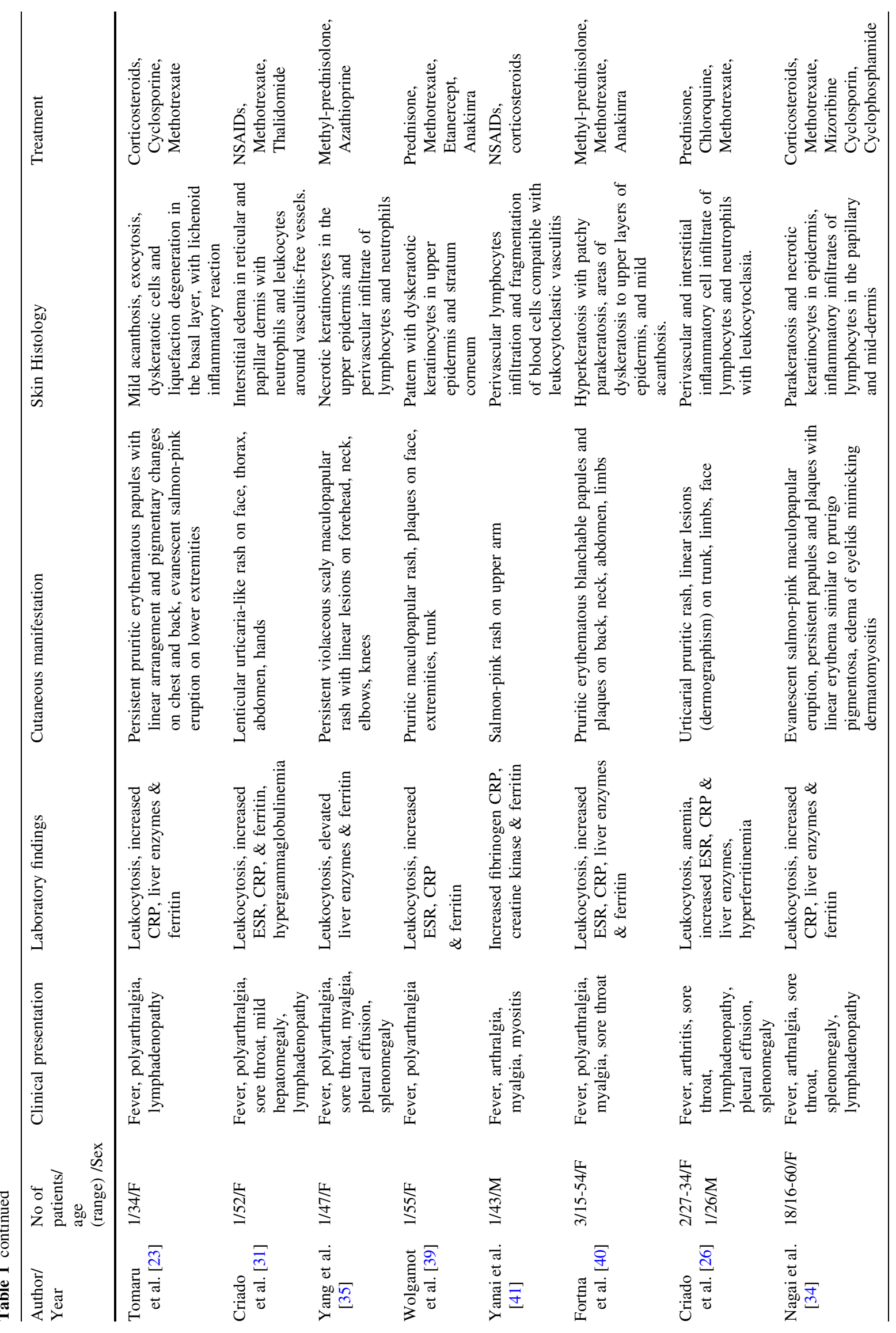




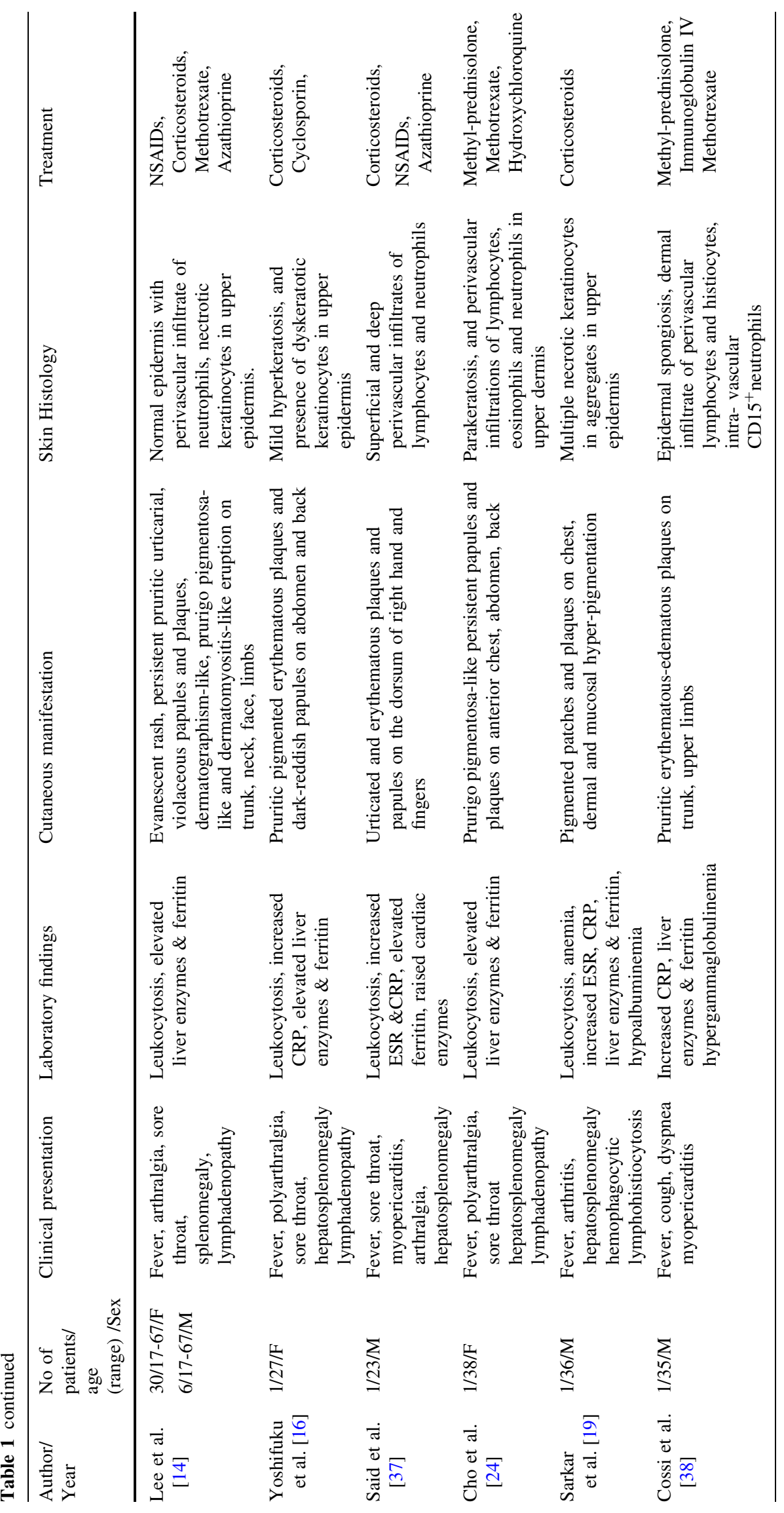


addition with increased production of IL-2, interferon- $\gamma$ and tumor necrosis factor (TNF)- $\alpha$. High levels of IL- 8 were demonstrated in Still's rash and this cytokine is considered as the inducer of the acute phase of inflammatory cascade in AOSD [32]. We believe that the cutaneous response to Anakinra was slower than the systemic one, since the febrile episodes resolved rapidly after initiation of Anakinra whereas the skin lesions persisted. The introduction of Anakinra therapy in our patient may have slightly exacerbated the pre-existing cutaneous lesions and caused new ones at the initiation of the treatment probably due to its immune modulating effects. Anakinra has been shown to cause interstitial granulomatous drug reaction $[33,34]$. The application of topical corticosteroids facilitated the resolution of the cutaneous manifestations.

The histological features of persistent eruptions include parakeratosis [35], necrotic keratinocytes in the upper epidermis and a perivascular and interstitial inflammatory infiltrate of lymphocytes and neutrophils in the upper- and mid-dermis [36-39]. In urticarial lesions the histopathologic findings demonstrate an intense infiltrate of mature $\mathrm{CD} 15_{+}$neutrophils between the dermal collagen bundles. This clinicopathological entity has recently been described as neutrophilic urticarial dermatosis (NUD) [40].

Dyskeratosis and dermal mucinosis represent distinctive cutaneous lesions of AOSD [41, 42]. The presence of fibrin thrombi in the small vessel with scarce inflammatory cell infiltration, suggestive of vasculopathy [25] has also been observed in cases of AOSD. Leukocytoclastic cutaneous vasculitis [43, 44] with mixed cryoglobulinemia [44] in AOSD has been described only rare in the literature.

Eosinophils that are commonly seen in drug-induced eruption are consistently lacking in the AOSD-associated skin lesions [14]. Perez et al. described a case of AOSDrelated persistent erythematous rash characterized by papules and plaques in which skin biopsy revealed perivascular infiltration of the small vessels in the dermis by eosinophils, histiocytes and lymphocytes [45]. In our case, the histopathology findings of the new persistent cutaneous eruption included the presence of several perivascular eosinophils not just in the dermis but also in the interstitium. The eosinophilic cutaneous manifestation could be either drug-related (i.e. antibiotics, Anakinra) or AOSD-associated.

Treatment of AOSD has been empirical. Non-steroidal anti-inflammatory drugs (NSAIDs), oral corticosteroids, disease modifying anti-rheumatic drugs (DMARDs), methotrexate (MTX), cyclosporine, azathioprine, sulfasalazine and minocycline have been used to treat this rare disease. Recently effective biologic agents including TNFa, IL-1 and IL-6 antagonist have been used for steroidresistant AOSD [46]. Laskari et al. provided evidence that Anakinra monotherapy or in combination with a DMARD, such as MTX may be the treatment of choice for patients with refractory Still's disease [47]. MTX is recommended in patients with polyarthritis and allows for steroid dose sparing in AOSD [48]. Our patient did not respond to corticosteroid treatment, but showed dramatic response to initiation of Anakinra treatment.

In summary, AOSD can manifest with atypical skin lesions that have highly distinctive but non-pathognomonic pathological features and are usually associated with severe disease. Still's rash can mimic various disorders with maculopapular, urticarial, linear and lichenoid manifestations and skin biopsy of those atypical cutaneous lesions is strongly recommended before or during the treatment course of AOSD because it allows rheumatologists and pathologists to recognize those specific distinctive histopathological characteristics and put the correct diagnosis.

Acknowledgments We thank Emeritus Professor H.M. Moutsopoulos, MD (Department of Pathophysiology, School of Medicine, National and Kapodistrian University of Athens) for his instructive comments and suggestions.

\section{Compliance with ethical standards}

Conflict of interest There is no conflict of interest. All authors participated in the preparation of this manuscript.

Human and animal rights For this case report formal consent is not required.

Informed consent Informed consent was obtained from all individual participants included in the study.

\section{References}

1. Fautrel B (2008) Adult-onset Still disease. Best Pract Res Clin Rheumatol 22(5):773-792

2. Yamagushi M, Ohta A, Tsunematsu T et al (1992) Preliminary criteria for classification of adult Still's disease. J Rheumatol 19:424-430

3. Appenzeller S, Castro GR, Costallat LT et al (2005) Adult onset Still disease in southeast Brazil. J Clin Rheumatol 11:76-80

4. Jaime MAI, Baptista R, Azevedo MNL et al (1998) Adult onset Still's disease: study on 25 cases. Rev Bras Rheumatol 38:285-290

5. Crispin JC, Martinez-Banos D, Alcocer-Varela J (2005) Adultonset Still disease as the cause of fever of unknown origin. Medicine (Baltimore) 84:331-337

6. Bywaters EG (1971) Still's disease in the adult. Ann Rheum Dis 30(2):121-133

7. Dino O, Provenzano G, Giannuoli G et al (1996) Fulminant hepatic failure in adult onset Still's disease. J Rheumatol 23(4):784-785

8. Hirohata S, Kamoshita H, Taketani T et al (1986) Adult Still's disease complicated with adult respiratory distress. Arch Intern Med 146(12):2409-2410

9. Vallianou NG, Kouvidou C, Naxaki A et al (2014) Acalculous cholecystitis with multiple organ failure and disseminated 
intravascular coagulation in a patient with adult onset Still's disease. Ann Gastroenterol 27(3):289-290

10. Bae CB, Jung JY, Kim HA et al (2015) Reactive hemophagocytic syndrome in adult-onset Still disease: clinical features, predictive factors, and prognosis in 21 patients. Medicine (Baltimore). 94(4): 451

11. Lee JY, Yang C, Hsu MM (2005) Histopathology of persistent papules and plaques in adult-onset Still's disease. J Am Acad Dermpath 52:1003-1008

12. Wouters JM, van Rijswijk MH, van de Putte LB (1985) Adult onset Still's disease in the elderly: a report of two cases. J Rheumatol 12(4):791-793

13. Koga T, Tokunaga N, Ichikawa $Y$ et al (1992) A 72-year-old female with adult Still's disease. Intern Med 31(12):1356-1358

14. Lee JY, Hsu CK, Liu MF et al (2012) Evanescent and persistent pruritic eruptions of adult-onset still disease: a clinical and pathologic study of 36 patients. Semin Arthritis Rheum 42(3):317-326

15. Kaur S, Bambery P, Dahr S (1994) Persistent dermal plaque lesions in adult onset Still's disease. Dermatology 188:241-242

16. Yoshifuku A, Kawai K, Kanekura T (2014) Adult-onset Still disease with peculiar persistent plaques and papules. Clin Exp Dermatol 39(4):503-505

17. Thien Huong NT, Pitche P, Minh Hoa T et al (2005) Persistent pigmented plaques in adult-onset Still's disease. Ann Dermatol Venereol. 132(8-9 Pt 1):693-696 (French)

18. Suzuki K, Kimura Y, Aoki M et al (2001) Persistent plaques and linear pigmentation in adult-onset Still's disease. Dermatology 202(4):333-335 (Review)

19. Sarkar RN, Bhattacharya R, Bhattacharyya K et al (2014) Adult onset Still's disease with persistent skin lesions complicated by secondary hemophagocytic lymphohistiocytosis. Int J Rheum Dis 17(1):118-121

20. Yammamoto M, Sueki H, Fujisawa R et al (1999) A case of adult Still's disease with atypical rash. Rinsho Derma 41:365-368

21. Phillips WG, Weller R, Handfield-Johnes SE et al (1994) Adult Still's disease. Br J Dermatol 130:511-513

22. Fujii K, Kobishi K, Kanno Y et al (2003) Persistent generalized erythema in adult-onset Still's disease. Int $\mathbf{J}$ Dermatol 42:824-825

23. Tomaru K, Nagai Y, Ohyama N et al (2006) Adult-onset Still's disease with prurigo pigmentosa-like skin eruption. J Dermatol 33(1):55-58

24. Cho YT, Liao YH (2014) Prurigo pigmentosa-like persistent papules and plaques in a patient with adult-onset Still's disease. Acta Derm Venereol 94(1):102-103

25. Lee JB, Kim JW, Lee SS et al (2002) Adult-onset Still's disease with vesiculopustules on the hands and feet. J Korean Med Sci 17(6):852-855

26. Criado PR, de Carvalho JF, Ayabe LA et al (2012) Urticaria and dermographism in patients with adult-onset Still's disease. Rheumatol Int 32(8):2551-2555

27. Tay YK, Paz RS, Ng SK et al (1996) A case of adult onset Still's disease presenting with fever and a rash. Ann Acad Med Singap 25(2):296-299

28. Soy M (2004) A case of adult-onset Still's disease presenting with angioedema. Clin Rheumatol 23:92

29. Peroni A, Colato C, Zanoni G et al (2010) Urticarial lesions: if not urticarial, what else? The differential diagnosis of urticaria: part II. Systemic diseases. J Am Acad Dermatol 62:557-570
30. Setterfield JE, Hughes GRV (1998) Kobza Black A. Urticaria as a presentation of adult Still's disease. Br J Dermatol 138:904-927

31. Criado RF, Criado PR, Vasconcellos C et al (2006) Urticaria as a cutaneous sign of adult-onset Still's disease. J Cutan Med Surg 10(2):99-103

32. Chen DY, Lan JL, Lin FJ et al (2004) Proinflammatory cytokine profiles in sera and pathological tissues of patients with active untreated adult onset Still's disease. J Rheumatol 31(11):2189-2198

33. Regula CG, Hennessy J, Clarke LE et al (2008) Interstitial granulomatous drug reaction to anakinra. J Am Acad Dermatol 59:S25-S27

34. Michailidou D, Voulgarelis M, Pikazis D (2014) Exacerbation of interstitial granulomatous dermatitis with arthritis by anakinra in a patient with diffuse large B-cell lymphoma. Clin Exp Rheumatol 32(2):259-261

35. Lübbe J, Hofer M, Chavaz P et al (1999) Adult-onset Still's disease with persistent plaques. Br J Dermatol 141(4):710-713

36. Nagai Y, Hasegawa M, Okada E et al (2012) Clinical follow-up study of adult-onset Still's disease. J Dermatol 39(11):898-901

37. Yang CC, Lee JY, Liu MF et al (2006) Adult-onset Still's disease with persistent skin eruption and fatal respiratory failure in a Taiwanese woman. Eur J Dermatol 16(5):593-594

38. Salaffi F, Filosa G, Bugatti L et al (2000) Urticaria as a presenting manifestation of adult-onset Still's disease. Clin Rheumatol 19(5):389-391

39. Said NH, Wong SN, Tan WH (2013) A case of adult-onset Still's disease presenting with urticated plaques and acute myopericarditis. Indian J Dermatol 58(5):405

40. Cozzi A, Papagrigoraki A, Biasi D et al (2014) Cutaneous manifestations of adult-onset Still's disease: a case report and review of literature. Clin Rheumatol. doi:10.1007/s10067-0142614-2

41. Wolgamot G, Yoo J, Hurst S et al (2007) Unique histopathologic findings in a patient with adult-onset Still disease. Am J Dermatopathol 29(2):194-196

42. Fortna RR, Gudjonsson JE, Seidel G et al (2010) Persistent pruritic papules and plaques: a characteristic histopathologic presentation seen in a subset of patients with adult-onset and juvenile Still's disease. J Cutan Pathol 37(9):932-937

43. Yanai H, Furutani N, Yoshida H et al (2009) Myositis, vasculitis, hepatic dysfunction in adult-onset Still's disease. Case Rep Med. 2009:504897

44. Elezoglou AV, Giamarelos-Bourboulis E, Katsilambros N et al (2003) Cutaneous vasculitis associated with mixed cryoglobulinemia in adult Still's disease. Clin Exp Rheumatol 21(3):405-406

45. Perez C, Montes M, Gallego M et al (2001) Atypical presentation of adult Still's disease with generalized rash and hyperferritinaemia. Br J Dermatol 145(1):187-188

46. Franchini S, Dagna L, Salvo F et al (2010) Efficacy of traditional and biologic agents in different clinical phenotypes of adult-onset Still's disease. Arthritis Rheum 62(8):2530-2535

47. Laskari K, Tzioufas AG, Moutsopoulos HM (2011) Efficacy and long-term follow-up of IL-1R inhibitor anakinra in adults with Still's disease: a case-series study. Arthritis Res Ther 13(3):R91

48. Fautrel B, Borget C, Rozenberg S et al (1999) Corticosteroid sparing effect of low dose methotrexate treatment in adult Still's disease. J Rheumatol 26(2):373-378 